\title{
ESTRATEGIAS GERENCIALES PARA EL MEJORAMIENTO DE LA GESTIÓN DEL TALENTO HUMANO Y RECURSOS DE LA ESCUELA.
}

\section{MANAGEMENT STRATEGIES FOR THE IMPROVEMENT OF HUMAN TALENT MANAGEMENT AND SCHOOL RESOURCES.}

\author{
Autora: Martha Nancy Mosquera Maldonado \\ E-mail de contacto: negra.mosquera@hotmail.com \\ ORCID ID: https://orcid.org/0000-0002-3976-3588 \\ Articulo recibido: 19 de Agosto del 2019 \\ Articulo revisado: 2 de Julio del 2019 \\ Articulo aprobado: 23 de Diciembre del 2019 \\ Licenciada en Ciencias de la Educación Mención Educación Primaria egresada de la Universidad de Guayaquil (Ecuador) \\ con 8 años de experiencia en la docencia. Posee una maestría en la Universidad Mayor San Marcos de Lima (Perú), mención \\ Gestión de la Educación.
}

\section{Resumen}

Este articulo científico presenta un análisis teórico que tiene como propósito conocer cuál es la relación entre las estrategias gerenciales y la gestión del talento humano y recursos dentro de una unidad educativa, de esa forma ayudar al personal directivo, docente y administrativo de la institución educativa a resolver algunas problemáticas que la organización ha venido arrastrando en materia de los recursos humanos, la presente investigación pone al alcance de los responsables de instituciones educativas, los resultados a los que se llegan, así como las conclusiones y sugerencias a fin de poner en práctica. De acuerdo con la problemática planteada se puede crear estrategias gerenciales para el mejoramiento de la gestión del talento humano y recursos de la escuela.

Palabras claves: Estrategias gerenciales, Gestión de talento humano, Institución educativa.

Abstract
This scientific article presents a theoretical
analysis that aims to know what is the
relationship between management strategies
and the management of human talent and
resources within an educational unit, thereby
helping the management, teaching and
administrative staff of the educational
institution to Solve some problems that the
organization has been dragging in human
resources, this research makes available to
those responsible for educational institutions,
the results that are reached, as well as the

conclusions and suggestions in order to implement. In accordance with the problems raised, management strategies can be created to improve the management of human talent and school resources.

Keywords: Management strategies, Human talent management, Educational institution.

\section{Sumário}

Este artigo científico apresenta uma análise teórica cujo objetivo é conhecer a relação entre estratégias de gestão e gestão de talentos e recursos humanos dentro de uma unidade educacional, auxiliando, assim, a equipe de gestão, ensino e administração da instituição de ensino. Para solucionar alguns problemas que a organização vem arrastando na área de recursos humanos, esta pesquisa coloca à disposição dos responsáveis pelas instituições de ensino os resultados alcançados, bem como as conclusões e sugestões para colocar em prática. De acordo com os problemas levantados, estratégias de gerenciamento podem ser criadas para melhorar o gerenciamento do talento humano e dos recursos da escola.

Palavras-chave: Estratégias de gestão, Gestão de talentos humanos, Instituição educacional.

\section{Introducción}

En la actualidad se viven momentos de cambios, propios de los procesos de la globalización. En el ámbito mundial y con exactitud en América Latina "muchas organizaciones ignoran que para ser 
competitivas y enfrentarse con éxito al cambio que nos empuja inexorablemente al mercado, han de saber motivar a su equipo humano". (Zobeida, 2015, pág. 16)

De allí pues, que la clave de la gestión del talento humano es importante en el éxito de los planes y logro de las metas organizacionales. Pues las instituciones que presenta diferentes problemáticas presentan desinterés en las actividades y por lo tanto poca motivación, estas instituciones presentan falencias en sus objetivos corporativos. Así mismo, las instituciones educativas son el reflejo de la sociedad siendo organizaciones complejas y sensibles a los problemas que puedan derivar.

En la actualidad es necesario que las instituciones se preocupen por conocer a sus miembros que trabajan y den lo mejor de sí, ya que el comportamiento de los que integran la organización trae como consecuencia los objetivos.

Efectivamente, el apoyo gerencial es indispensable para un buen funcionamiento educativo, donde: La gestión educativa debe plantear seriamente su transformación, donde el principio que rija su funcionamiento debe ser el uso de estrategias de alta gerencia, lo que implica gerentes proactivos y creativos que permitan la participación de todos los sectores que integran la organización, bajo la filosofía de equipos de alto desempeño. (Martínez, 2013, pág. 1)

En la actualidad, a nivel internacional, las instituciones educativas se distinguen por presentar falencias en la parte organizativa, ello ha producido un colapso ante los cambios apremiantes en diversos ámbitos y esto involucra que hay retos para democratizar el conocimiento científico, cultural, económico y ambiental, ante esta perspectiva, el directivo como gerente pedagógico debe constituirse en protagonista de los cambios educativos y organizacionales. Las instituciones educativas son vistas como organizaciones formales, complejas y abiertas que presentan características peculiares, pero que comparten rasgos comunes en todos los aspectos, en el sentido de que incorporan energía del ambiente, procesa o transforman esa energía, la convierten en un sistema capaz de adaptarse a la organización.

Las estrategias gerenciales son aquellas que se emplean en una organización para lograr resultados positivos. En la institución educativa, se utilizan para el progreso de los aprendizajes de los educandos, como producto de una buena gestión escolar, de la apropiada administración de los recursos: humanos, materiales y financieros, entre otros.

Al respecto, Chiavenato (2009:454), destaca que las estrategias gerenciales, se refieren " al comportamiento de la organización como un todo integrado, es holística, sistemática y medular, donde se planea, calcula y se asigna recursos a las actividades básicas para el éxito de la empresa", es decir, es una postura que adoptan las organizaciones para administrar las relaciones institucionales y su entorno, las cuales se organizan de manera estratégica y organizada en función de lograr los objetivos y metas establecidas en la institución.

De allí que la acción gerencial ha de convertirse en el proceso innovador para poder lograr objetivos, que anticipen y respondan a los cambios que ciertamente, pues solo a través de la educación es posible el logro de todos los retos que se le presentan en el ámbito educativo. Para ello es necesario, profundizar los conocimientos para poder ejercer las funciones básicas gerenciales ya que indudablemente el desempeño del gerente educativo requiere de competencias profesionales, habilidades, destrezas, como también formación de valores que le permitan una efectiva integración y 
participación ciudadana en la organización, la cual ha de ser precedida por directivos $y$ docentes, con verdadera vocación de servicio, y sobre todo de compromiso.

Por lo tanto, se requiere de un gerente competente que maneje adecuadamente las estrategias gerenciales inherentes a su cargo, definidas para este mismo autor, como el proceso mediante el cual se toman las decisiones (conscientes e intencionales), que necesitan manejarse de manera coordinada, con los debidos conocimientos para poder cumplir una determinada demanda u objetivo, dependiendo de las características de la situación educativa en que se produce la acción. Desde esta perspectiva, las estrategias gerenciales se conviertan en la capacidad estratégica de un directivo y su relación con el contexto de la organización, considerando que a través de éstas estudie el entorno y los procesos internos de la institución tanto integradoras como motivacionales, a fin de obtener al máximo la calidad de desempeño en la institución.

\section{Desarrollo}

\section{Estrategias gerenciales}

Etimológicamente a palabra "estrategia es de origen griego. Estrategia: Estratego o el arte del general en guerra, procedente de la fusión: stratos (ejército) y agein (conducir, guiar)". (Rojas, 2015, pág. 34)

De manera que, estrategia proviene del ámbito militar entendida como el arte de dirigir operaciones militares. Una estrategia es un conjunto de acciones que conllevan a resolver problemas para un resultado óptimo. Por su parte, Fiallos (2014) menciona que:

La estrategia como un medio de establecer el propósito de la organización. Esto es en términos de sus objetivos a largo plazo, la estrategia es un modo de dar explícitamente forma a las metas y los objetivos de la organización definiendo los principales programas de acción.

En este sentido, la estrategia tiene una intención que va dirigida hacia un objetivo, en el campo de una institución va dirigida a metas bien definidas. Agregando a estas ideas, Contreras (2013) considera que:

La estrategia se constituye en un aspecto muy importante en las decisiones que deben tomar las personas que tienen a cargo la gestión de una organización, en la que hay recursos de todo tipo que deben ser utilizados en forma óptima para cumplir con las políticas y metas trazadas. (pág. 153)

De allí, se aborda que las estrategias son un factor clave en la gerencia organizacional para cumplir con los objetivos que se han propuesto. En este sentido las estrategias gerenciales son acciones enfocadas a orientar y evaluar las acciones de la institución para que logren metas. Además," es importante destacar que las estrategias estarán siempre en función del tipo de organización, tomando en consideración tanto aspectos internos como externos de la organización”. (Peraza, 2012, pág. 89)

De acuerdo con Parra (2009) define las estrategias gerenciales como:

"La formulación, ejecución y evaluación de acciones que facilitarán el logro de los objetivos organizacionales. Corresponde a un enfoque objetivo y sistemático para la acción que permite la organización y utilización de información cualitativa y cuantitativa para la toma de decisiones efectivas en circunstancias de incertidumbre. (pág. 36)"

Entonces las estrategias gerenciales ayudan a optimizar a las instituciones, afrontando retos, 


\section{Ciencia y Educación (ISSN 2707-3378) \\ Vol. 1 No. 3 \\ Marzo del 2020}

donde las decisiones determinen el futuro y fortaleciendo la gestión, cabe resaltar que para "proponer enfoques estratégicos audaces y paradigmáticos caracterizados por la transformación e innovación, donde el directivo, coordinadores supervisores constantemente tienen que explorar y analizar las nuevas tendencias educativas para lograr la transformación”. (Hernández, 2013, pág. 37)

En este orden de ideas, es importante resaltar el apoyo de los miembros de la institución y tengan esa calidad de compromiso. A esto se suma la importancia de las estrategias gerenciales, expuesto por Bonilla (2013):

- Mejora el proceso de decisión.

- Ayuda a identificar y entender los principales temas.

- Ayuda a administrar la complejidad.

- Acrecienta la flexibilidad e innovación.

Serna (2003) definió "Estrategias gerenciales son aquellas actividades que permiten alcanzar o realizar un proyecto, para tener la eficacia en la planeación, organización, dirección y control de la gestión estratégica" (p.246).

La estrategia gerencial es importante para cualquier institución grande o pequeña, es necesario que se den excelentes decisiones para llevar a la institución directo a sus objetivos y al acatamiento de su misión y visión. Entonces podemos entender que la estrategia gerencial se perfila en una institución con la finalidad de impulsar de manera efectiva los cambios y enfrentar los desafíos que le propone el contexto, consiste en una serie de técnicas utilizadas por el gerente para alcanzar que los objetivos de la institución se logren con efectividad y eficiencia.

Igualmente, Cortazar (2007) destacó: Estrategia gerencial es un modelo personal de procesos de gestión en la que una institución desarrolla acciones específicas en un contexto determinado con la finalidad de lograr los propósitos que se ha trazado. Son normas eficientes de acción, entre ellas se encuentran la toma de decisiones, la planificación, el liderazgo y la comunicación (p. 131)

Según González y Pelekais (2010) “las estrategias gerenciales constituyen una herramienta fundamental en materia organizacional para el próspero desarrollo de las organizaciones, ya sean éstas de carácter público o privado" (p.343). Por ello, las estrategias gerenciales son herramientas aplicadas por el líder de una institución para el logro y perfeccionamiento de la gestión, finalmente un líder debe incrementar formulas apropiadas para que la institución sea exitosa.

Desde esa perspectiva, Ramos (2007) señaló: "el gerente pedagógico debe coordinar la labor que realiza y así desarrollar una estructura sólida, encomendando responsabilidades con la autoridad correspondiente e instituir fluidas relaciones proporcionando el alcance de los objetivos de la institución" (p.34), En ese sentido, el directivo o jefe se concibe ahora desde una visión gerencial, ya que agrupa las condiciones indispensables para conseguir de los docentes, el desempeño integral $y$ placentero, de acuerdo con las demandas.

\section{Tipos de estrategias gerenciales}

Hernández (2013, págs. 39-53) menciona los siguientes tipos de estrategias gerenciales:

- Estrategias de enlace

Una institución educativa, como organización no puede dejar por sentado, los compromisos con otras instituciones, al igual que con la sociedad en general, por lo que debe elaborar, revisar y ejecutar periódicamente un plan de trabajo coordinado con cada actor como 
también con los organismos involucrados para activar las estrategias que se han de seguir.

Por su parte, Cardona (2006), manifiesta que el director, coordinador o supervisor también debe favorecer el enlace en el proceso de integración entre docentes, padres, representantes e institución educativa, con el propósito de establecer las estrategias de interacción comunicacional a fin de fortalecer los problemas educativos de acción pedagógica, conocer las debilidades y fortalezas sobre el proceso de enseñanza aprendizaje de sus hijos, así como también los problema del entorno socio cultural comunitario, entre otros. Asimismo, señala, que la integración es la participación continua y directa de los padres, representantes y adultos significativos para estar inmersos dentro de las responsabilidades o finalidades del proceso educativo.

\section{- Estrategias de delegación}

Puede verse como los procesos de coordinación, comunicación concertación y acciones tendientes a buscar enlaces y mejorar las relaciones interpersonales e interinstitucionales desarrolladas por los miembros de la institución, así como también coordinar esfuerzos para integrar a los padres, representantes y comunidad en general a las actividades educativas. En este proceso, el personal directivo debe tener una comunicación interfuncional como la capacidad de conocer la institución más allá de los límites de la propia función, para comprender la interrelación en distintas unidades y desarrollar la cooperación de todos los actores que hacen vida en el hecho educativo

Al respecto, Pérez (2005:78), las define como "la capacidad de conseguir que los colaboradores del equipo dispongan de la información e igualmente, los recursos necesarios para tomar decisiones para lograr sus objetivos; además de asignarles responsabilidades". Para el autor antes citado, las actitudes personales de quien dirige son sumamente importantes, entre ellas la receptividad del gerente para tener la disposición a conceder que otras personas pongan en práctica sus ideas, de colaborar con ellos a la vez elogiar su inventiva; entre otras esta, la disposición a ceder o delegar en sus subordinados el derecho a tomar sus decisiones, adicionalmente, el ser flexible para que otros puedan cometer errores además de confiar en todos los miembros del grupo estableciendo y aplicando controles que permitan revisar constantemente los procesos para no perder el control.

Según Chiavenato (2009), la situación delegativa por la concepción de dirección administrativa, y mejor aún por la de liderazgo, viene también a hacer hincapié en, que para conducir o motivar a la gente, se requiere algo más que manejarla; se necesitan conceptos básicos sobre las potencialidades y atributos del subordinado. En este sentido, la acción gerencial así entendida, se basa en que la gente quiere trabajar. Por lo tanto, no se puede suponer que no quiere laborar, ya que según este autor, la mayor parte de la gente se desintegra física y moralmente sino trabaja.

Para Daft y Marcic (2005:115), "se refiere a la capacidad de encargar a otras personas de la realización de tareas para un propósito grupal; significa asignar responsabilidad y autoridad de tareas para un propósito grupal; significa asignar responsabilidad y autoridad en subordinados a fin de lograr una mayor fluidez en los procesos de trabajo". Es decir, la capacidad de conseguir que el equipo docente dispongan de la autoridad para dar información y los recursos necesarios para tomar decisiones, e igualmente lograr los objetivos; además de asignarles responsabilidades. 
Por su parte, Soto (2006), señala que, representa la asignación de funciones tanto administrativas como gerenciales en el personal, la cual debe ser de manera democrática y funcional, al proporcionar lineamientos generales para la ejecución de tareas específicas, luego se dedican a la observación, verificación para hacer simples sugerencias. Sin embargo, el gerente educativo continuará siendo el responsable por el resultado de las decisiones. Dentro de este aspecto, se resaltan aquellas capacidades que presenta el directivo las cuales resaltan su experiencia en el ámbito de su desempeño.

En pocas palabras la delegación se refiere a la capacidad de encargar a otras personas de la realización de tareas para un propósito grupal; significa asignar responsabilidad y autoridad en subordinados a fin de lograr una mayor fluidez en los procesos de trabajo. Es decir, la capacidad de conseguir que los colaboradores del equipo dispongan de la información y los recursos necesarios para tomar decisiones y lograr sus objetivos; además de asignarles responsabilidades

\section{- $\quad$ Estrategias de gestión}

La función básica del gerente educativo, es la de lograr el mejoramiento de los resultados del proceso de enseñanza, es decir, lograr que se mejore cada vez más la calidad del producto final del mencionado proceso, de forma orgánica, sistemática, organizada y continua, a lo largo del proceso educativo, sobre toda de la acción gerencial, pues es allí donde se van utilizando adecuadamente los recursos humanos y materiales para alcanzar en forma eficiente los objetivos educativos y para ello es necesario que el directivo presente un estilo o manera de gerenciar lo más ideal que sea posible para mejorar e implementar las estrategias de gestión.
Pérez (2005:142), las define como "la actividad de dirigir, en un nivel organizado, en los casos en que los miembros componentes o no del grupo de la gerencia de una institución se encuentran en contacto". Es decir, representan las acciones que conducen a la coordinación de actividades en pro de las metas institucionales.

De acuerdo con los criterios de estos autores, las estrategias de gestión gerencial pueden ser vistas como el servicio especializado en el cual no puede improvisarse el material humano, cuyo fin primordial consiste en mejorar los factores y condiciones en que se realice el proceso enseñanza - aprendizaje, como también administrativo. Por lo tanto, el eficaz desempeño del recurso humano constituye una de las claves del éxito de toda institución.

Por ello, es necesario la evaluación de los individuos que desempeñan diferentes roles dentro de la organización, en especial el gerente debe determinar su aporte a la eficiencia organizacional; en consecuencia, el gerente educativo, debe contemplar la realidad de cada escuela, el conjunto del sistema escolar, en relación a las necesidades y aspiraciones sociales, el gerente no es tan sólo un observador escolar, un pedagogo capacitado para difundir innovaciones educativas, para orientar sobre métodos didácticos o diseñar programas concretos de actuación en la medida del centro escolar.

Ahora bien, para que el eficaz desempeño sea efectivo y exitoso la organización debe estar dirigida por un gerente que reúna un perfil calificado que tenga la adecuada formación profesional para poder ejercer las funciones administrativas y procedimentales de manera ideal. Al respecto, la formación del gerente educativo, según Planas (2002:38), “es un elemento fundamental que contribuye al desempeño óptimo de los individuos dentro de una organización". 
En virtud de esto, la institución debe velar por el adiestramiento en las labores a ejecutar por los directivos y docentes, para contribuir al logro de los objetivos, a través de estudios de mejoramiento profesional, seminarios, talleres, foros, conferencias, entre otros los cuales le permitan obtener el perfil requerido de acuerdo con las tareas o funciones específicas de cada cargo a ocupar.

Cada acción de la actividad gerencial requiere del directivo, la planificación acciones para prever el futuro de la organización, como un ordenamiento racional de los recursos, como también de los objetivos precisos que persigue, lo cual implica una formulación y ejecución de estrategias, las cuales se definen y organizan a través de un conjunto de procesos coherentes y relacionados que abarca desde los objetivos como también propósitos generales, hasta la acción más detallada del quehacer pedagógico en el proceso organizacional.

Al respecto, Chiavenato (2009), plantea que para administrar la interdependencia entre elementos del ambiente de trabajo, cada organización utiliza estrategias distintas, dependiendo de cada situación en particular, ello implica coacción y contingencias, de tal forma requiere la suma de todos los miembros de la organización, para aumentar el poder de la organización y reducir su independencia.

Esto significa, que en la medida que el gerente defina la estrategia a seguir para el logro de los objetivos y establezca comportamientos, planificaciones y sistemas administrativos de apoyo, el personal directivo dispondrá de una orientación y autentica acción gerencial para encaminar sus esfuerzos e integrar todo el personal, además la comunidad hacia los procesos responsables en pro de la unificación de criterios para poder lograr la interdependencia.
Según Robbins (2005:101), una auténtica acción gerencial, supone la revisión exhaustiva del proceso y de la institución, si no se transforma en un simple programa de instrucción; en suma la intencionalidad es mejorar la calidad del servicio educativo.

Al respecto, es preciso revisar la definición expuesta por este autor sobre la estrategia, quien la define como "una herramienta que ayuda a los administradores a desarrollar nuevas aptitudes y procedimientos para encarar los retos frente a los cambios para lograr la calidad de desempeño".

En este contexto, las estratégicas gerenciales involucran las acciones para afrontar los retos ante las innovaciones, estas pueden ser desarrolladas de diversos tipos por parte de los gerentes educativos para promover los cambios que sean necesarios en el ámbito escolar, conformando equipos de trabajo de alto desempeño, el sentido de pertenencia y revisión permanente de la cultura escolar respecto al tipo de escuela que quiere ser, según sea el fin o propósito que se pretende lograr.

En referencia a los tipos de estrategias Chiavenato (2009), destaca las de adaptación, cooptación, coalición, competencia; por otro lado plantea otras las estrategias organizacionales con sus repercusiones en los tres niveles administrativos estas están relacionadas con las defensivas, ofensivas, analítica y reactiva, todas ellas fundamentan acciones, en el marco de la gerencia de las organizaciones, su implementación es la suma total de las actividades al igual que de las decisiones necesarias para ejecutar un plan estratégico. Es decir, el proceso que se utiliza para poner en práctica las políticas y lineamientos organizacionales 


\section{Dimensiones de Estrategias gerenciales}

Dimensión 1: Planeamiento estratégico

En esta fase el gerente y/o líder pedagógico, resuelve qué y cómo hacer, para transformar la institución en una entidad de excelencia, de acuerdo a los propósitos planteados en el Proyecto educativo Institucional. En este orden de ideas. Serna (2003) formuló:

La estratégica ideal consiste en seleccionar los proyectos estratégicos que son la clave del éxito de la institución y debe tener un desempeño excepcional como condición para lograr los objetivos, la misión y visión. Un plan es estratégico cuando es claro, flexible, único y se realiza a corto plazo. (p. 243)

En este sentido Mojica y Rodríguez (2012) refirieron: Planificar implica definir objetivos o metas, se planea por tres razones: (a) fijar el futuro de la institución, (b) identificar y asignar los recursos que necesita y (c) designar las actividades necesarias.

La planificación como estrategia para la superación persistente, Manes (2003) consideró como exigencias fundamentales:

“(a) es posible mejorar la gestión institucional y de ello el director debe estar convencido; (b) la ruta para la mejorar es propiciar una gestión participativa; (c) las personas que anteponen sus beneficios no son idóneos para lograr los propósitos de mejoría incesante; (d) el proceso de mejora debe instalarse en la organización, considerando las ventajas convenidas para mantener los modelos de calidad; (e) la prevención debe ser compromiso de todos los integrantes de la institución enfocado a mejorar la productividad. (p. 49)."
Dimensión 2: Organización estratégica

La organización estratégica está creada para alcanzar metas.

Desde esta perspectiva, Serna (2003) formuló: Para hacer las cosas en todas las áreas "Debemos modernizarnos tecnológicamente" (p. 244). Esta estrategia permite analizar diferentes propuestas con claridad y concretizar los objetivos de la gestión educativa. Igualmente nos permite entender la importancia de trabajo e innovación en los diferentes campos de la actividad humana.

Ante la situación planteada, Mojica y Rodríguez (2012) indicaron: La organización consiste en la agrupación, distribución y coordinación de las tareas. Una organización es eficaz cuando el director coordina mejor los recursos humanos, materiales y de información.

Chiavenato (2001) definió: “Organización es establecer actividades concretas para alcanzar los objetivos trazados y agrupar las tareas a personas específicas" (p. 256).

Amarante (2000) sostuvo:

"La organización involucra el diseño de la estructura formal para el progreso de la administración pedagógica, proporcionando la integración y coordinación de las actividades de los agentes educativos; y el uso de los recursos para desarrollar los programas, proyectos y procesos que comprenden la distribución del trabajo y de funciones, por medio de una jerarquía de mando y compromiso de sus actores. (p. 78)."

Dimensión 3: Dirección estratégica

La dirección estratégica es una herramienta que pone en práctica las potencialidades de una institución educativa. En este sentido, Serna 
(2003) formuló: "Debemos estimular la participación y la capacidad de nuestros talentos" (p. 244). Para materializar este trabajo de investigación e innovación se debe determinar el perfil y las funciones del director $\mathrm{y}$ los docentes en general.

Mojica y Rodríguez (2012) afirmaron: La dirección estratégica envuelve los conceptos de motivación, liderazgo, guía, estímulo y actuación. Es el resultado del esfuerzo de cada miembro de la institución educativa que logra cumplir con sus objetivos de forma óptima.

La dirección estratégica aunada con el liderazgo, la motivación y la innovación de un clima organizacional por parte del director, "integra las potencialidades de los diferentes sujetos, a partir del compromiso de todos con el proyecto para mejorar el servicio y la administración de los recursos de la organización" (Graffe, 2000, p. 75).

Dimensión 4: Control estratégico

En la última fase del proceso administrativo se miden los resultados obtenidos. Según Mojica y Rodríguez (2012) concluyeron: Control es medir cuantitativa y cualitativamente la ejecución y determinar acciones correctivas que encauce la ejecución.

Ante los nuevos retos y desafíos educativos para los directores del siglo XXI, se propone una cuarta estrategia para elevar la calidad educativa y el logro escolar con principio de equidad. En relación con lo anterior, Serna (2003) formuló: "Elaborar planes de ajuste que deben tener seguimiento, monitoreo $\mathrm{y}$ evaluación" (p. 256, 257). En definitiva, si se planea, se organiza y cada persona, comité o área operativa implementa sus actividades de manera aislada, fuera de contexto, tratando de desarrollar por separado pequeñas tareas que no fueron acordadas, seguramente serán agotadoras y no facilitan el proceso, nunca se verán los resultados anhelados por el colectivo y la estrategia fallará.

\section{Gestión del talento humano}

Para abordar de manera puntual este concepto gestión del talento humano, es importante indicar las siguientes definiciones que se ha creído pertinente por los distintos autores:

Huergo (s.f., pág. 1) señala que "la palabra gestión proviene de "gestus", una palabra latina que significa: actitud, gesto, movimiento del cuerpo". A mitad del siglo XX la gestión surge como disciplina como una evolución administrativa moderna "en su desarrollo, las definiciones de gestión se han elaborado a partir de criterios tales como: el objeto del cual se ocupa, los procesos que intervienen en ella, la organización de recursos, los objetivos y la interacción entre personas". (Correa, Álvarez, \& Correa, s.f., pág. 8)

Por su parte, Garzón (2011, pág. 11) “llama gestión (en palabras propias) a aquellas acciones relacionadas con actividades que están dirigidas a la realización y desarrollo de objetivos que han debido establecerse con anterioridad".

Se concibe que la gestión son las responsabilidades sobre un determinado proceso que pueden estar orientadas a las actividades correspondientes.

\section{Talento humano}

El talento se caracteriza por varios aspectos o cualidades de un individuo relacionada con las habilidades excepcionales, la cual implica conocimiento (lo que se conoce como competencia cognitiva), compromiso (personalidad y esfuerzo) y poder (valores y decisión). 
Jericó citado Prieto (2013, pág. 17) define el talento:

Como aquella gente cuyas capacidades están comprometidas a hacer cosas que mejoren los resultados en la organización. Asimismo, define al profesional con talento como un profesional comprometido que pone en práctica sus capacidades para obtener resultados superiores en su entorno y organización.

En cuanto a las definiciones que se orienta a la gestión del talento humano, a esto suman los criterios de atraer y motivar a los profesionales más competencias en una empresa, donde la persona pone en práctica sus capacidades en una organización determinada y obtener resultados óptimos.

Por su parte, Jaramillo (2005, pág. 107) define que la gestión del talento humano es el conjunto de procesos necesarios para dirigir a las personas o recursos humanos dentro de la empresa, partiendo del reclutamiento, selección, capacitación, recompensas, evaluación del desempeño, salud ocupacional y bienestar, entre otros, que conduzcan a la obtención de valor agregado para la empresa, los empleados y el entorno.

\section{Objetivos de la gestión del talento humano}

Martínez (2013) sostiene que el factor humano cumple un rol activo en las diferentes organizaciones, por ello si las personas pueden aumentar o disminuir sus fortalezas tendrá repercusión en la eficiencia de la organización. Por ello es necesario que los trabajadores de la organización asuman un compromiso lo cual le servirá para ayudar a que la organización alce su objetivo, proporciona competitividad a la organización. Suministra a la organización empleados bien entrenados y motivados, permitir el aumento de la autorrealización y la satisfacción de los empleados en el trabajo, desarrollar y mantener la calidad de vida en el trabajo, administrar el cambio, establecer políticas éticas y desarrollar comportamientos socialmente responsables (p.58)

Chiavenato (2002) menciono que las finalidades de la gestión de personas deben favorecer a la eficiencia de la organización mediante el apoyo para que la organización alcance sus objetivos, brindar a la institución empleados bien entrenados y motivados, todo ello gira entorno a definir cuáles son las necesidades de la organización

\section{Procesos de la gestión del talento humano}

Chiavenato (2002) menciono que existen seis procesos, el primero es la admisión de personas que hace referencia a los procesos utilizados para ingresar nuevos trabajadores a la empresa, la adaptación de personas que refiere a procesos en los cuales se diseñan actividades que van a realizar los trabajadores.

La compensación de personas son procesos que se utilizan para generar recompensas e incentivos y satisfacer sus necesidades, el desarrollo de las personas en el cual se capacita a los empleados para logra mayor productividad en ellos.

El mantenimiento de personas alude a procesos que sirven para crear las condiciones adecuadas tanto físicas como psicológicas para los trabajadores, el monitoreo de personas refiere a procesos que se basan en el acompañamiento y control de lo realizado por las personas así como constatar los resultados obtenidos

\section{Funciones de la gestión del talento humano}

Chiavenato (2009) sostuvo que está constituida por las personas y las organizaciones, por ello se considera como un área de bastante sensibilidad en la cual el autor señala que se deben considerar los siguientes elementos: 
La planificación de necesidad de personal, por ello es necesario conocer primero que personas necesita la empresa, esta planificación es de importancia para las empresas ya que les permitirá asignar el número de personas que necesita la empresa para evitar a posteriores despidos.

El análisis y descripción de puestos de trabajo está referido al proceso de determinar las habilidades y conocimientos previos que se necesitan para determinados puestos, lo cual debe ser de conocimiento a los trabajadores. La administración de compensaciones el cual abarca aspectos de valoración del puesto el cual servirá en el proceso de generar las remuneraciones respectivas, si la compensación de los trabajadores no es satisfactoria esto generara que con facilidad abandonen el trabajo.

El reclutamiento y selección de personal en primer lugar se realiza el reclutamiento en el cual es importante planificar y sistematizarlo, en cuanto a la elección se realizará del grupo que fue reclutado, por lo cual ambos procesos son integrados

La inducción del personal al cual también se le conoce como proceso de adaptación, orientación del nuevo personal con la intención de darle una visión general de la empresa. La capacitación y desarrollo está referida a las actividades de capacitación, que buscan no solo el desarrollo personal sino también de la organización, hay que tener presente que este debe darse de manera constante para mejorar la productividad.

El plan de desarrollo de carrera profesional el cual no solo hace referencia a adquirir conocimientos y habilidades para un determinado puesto, sino que se busca que el empleado logre el éxito. La evaluación del desempeño la cual es un proceso para calificar, estimular las cualidades de las personas lo cual debe darse periódicamente, la cual puede darse por merito o por actuación.

La comunicación interna hace referencia a las formas que tiene la empresa de enviar información al exterior. La motivación laboral la cual puede ser externa o interna, las cuales debe ser considerada por las empresas.

La dirección de equipos de trabajo en la cual se permite que los trabajadores también tomen decisiones en beneficio de la empresa Robbins y Judge (2009) señalaron que los esfuerzos que se realizan individualmente generan un desempeño mayor si se trabaja en equipo

El clima laboral la empresa debe hacer lo posible para que el tiempo que pasa el trabajador en ella se tenga un clima atractivo. La cultura de la organizacional fomenta le compromiso con la institución y como el trabajo de cada persona es beneficioso para toda la institución.

No se debe dejar de lado que las personas están en constante cambios por eso se habla de la gestión del cambio si esto es necesario realizar se debe empezar con un piloto para analizar los resultados y después ser aplicados a toda la organización

La gestión del tiempo, toda decisión de renovación necesita que las personas claves dediquen horas en diligencias como: razonamiento, planeamiento, tarea en contribución y entrenamiento. El liderazgo organizacional en la cual se necesita que sean lideres auténticos, finalmente se señala la gestión del estresen

Dimensiones de la gestión del talento humano

Chiavenato, (2009) menciono que los procedimientos para detener a los colaboradores están constituidos por las vínculos con los 
trabajadores, limpieza, seguridad y condición de vida.

Recompensar a las personas, Chiavenato, (2009), explica que "los procesos para recompensar a las personas constituyen los elementos fundamentales para incentivar $\mathrm{y}$ motivar a los trabajadores de la organización, siempre que los objetivos organizacionales sean alcanzados y los objetivos individuales sean satisfechos" (p. 276).

\section{Desarrollo de las personas}

Chiavenato (2009) mencionó que en este caso no es solo darle a la persona abundante información para que conozca nuevos conocimientos, sino que todo ello le sirva para cambiar costumbres y conductas, de esta manera sean más eficaces en la labor que realizan

Retener a las personas, Chiavenato, (2009) sustenta que los métodos para inmovilizar a las individuos está constituido por los vínculos con los empleados, pulcritud, seguridad y calidad de vida.

Supervisar a las personas, Chiavenato, (2009) mencionó que: "la palabra supervisar adquiere un significado especial en esta parte, que es seguir, acompañar, orientar y mantener el comportamiento de las personas dentro de determinados límites de variación" (p. 504).

\section{Conclusión}

Se determino mediante este estudio que las estrategias gerenciales son el proceso de coordinación de los recursos para el logro de un objetivo presente en todos los ámbitos de la vida humana y el hecho educativo no escapa a esta realidad. Si bien es cierto que en un principio la gerencia estuvo encasillada solo dentro de las organizaciones privadas que tenían metas comerciales el transcurrir de los años ha permitido entender que la gerencia permite el desarrollo eficiente de proyectos en cualquier ámbito de la vida.

Atendiendo a esto se ha desarrollado la Gerencia Educativa que es una herramienta fundamental para el logro y funcionamiento efectivo de la estructura educativa, Alarcón (2013) afirma que la gerencia educativa es un proceso de coordinación de una institución educativa por medio del ejercicio de habilidades directivas encaminadas a planificar, organizar, coordinar y evaluar, la gestión estratégica de aquellas actividades requeridas para alcanzar la eficacia pedagógica, la eficiencia administrativa." la gerencia educativa es el proceso de organización y empleo de recursos para lograr los objetivos preestablecidos a través de una eficiente organización donde el gerente educativo debe dirigir su equipo hacia el logro de los objetivos.

La importancia de la gerencia educativa radica en que proporciona las bases para encaminar los procesos que permitirán desarrollar proyectos, atendiendo a los recursos con los que se cuenta dentro de la institución educativa y a las variables ambientales, sociales, económicas y humanas que entran en juego en todo proceso gerencial. El gerente deberá tener no solo un formación gerencial, sino humana que le permita entenderse y manejarse dentro de las variables que se presenten en el entorno, sin dejar de lado el objetivo planteado y logrando subsanar los imprevistos que se presenten a lo largo del camino.

La Gerencia Educativa debe garantizar el desarrollo de la calidad educativa gestionando aspectos tales como: disponer de las herramientas necesarias para lograr la eficacia institucional, tomar decisiones de índole administrativas, presupuestaria, pedagógicas y trabajar con indicadores claves de eficacia y efectividad para medir a toda la organización 
educativa. Sera entonces la gerencia educativa la que permita que las instituciones logren llegar a la excelencia atendiendo a las necesidades de la población.

\section{Referencias Bibliográficas}

Alarcón, S. (2013). Gestión educativa y calidad de la educación en instituciones privadas en Lima Metropolitana. (Tesis de maestría) Lima: Universidad San Martín de Porres.

Bain. (2003). La productividad. Colombia: McGraw-Hill.

Barreto, C. (2012). Evaluación de la calidad del desempeño docente y directivo en el Instituto Superior Agropecuario José Benigno Iglesias, de la parroquia Biblián, del cantón Biblián, de la provincia del Cañar, durante el año académico 2011-2012. (Tesis de maestría) Loja: Universidad Técnica Particular de Loja.

Beltrán, F. (2007). Política versus gestión escolar. Revista Novedades Educativas, 18.

Bittel. (2000). Administración de Personal.

Chiavenato, I. (2000). Administración de Recursos Humanos. Colombia: McGrawHill.

Chiavenato, I. (2011). Administración de recursos humanos. El capital humano de las organizaciones. México: McGraw-Hill Interamericana.

Da Silva, R., \& Reinaldo, O. (Enero de 2008). Administración. Obtenido

de http://www.promonegocios.net/administraci on/definicion-eficacia.html.

Druker, P. (2002). Los desafíos de la gerencia del siglo XXI. Bogotá: Norma.

Galindo, C. (2006). Manual para la creación de empresas: Guía de planes de negocios. Bogotá: ECOE.

González, A. (2013). Liderazgo del director para el desempeño laboral de los docentes en la U.E.E. José Tadeo Monagas. (Tesis de maestría) Venezuela: Universidad Santa María.

González-Palma, J. (2005). De la gestión pedagógica a la gestión educativa. Una tarea inconclusa. Observatorio ciudadano de la educación. (Vol. V). Obtenido de http://www.observatorio.org/colaboraciones /gonzalezpalma2.html

Instituto Peruano de Evaluación, Acreditación y Certificación de la Calidad de la Educación Básica. (2011). Matriz de Evaluación para la acreditación de la calidad de la Gestión Educativa de Instituciones de Educación Básica Regular. Obtenido de http://www.ipeba.gob.pe/images/stories/MA TRIZ_DE_EVALUACION_PARA_ACRE DITACION_3.pdf

Kootz, H., \& Weihrich, H. (2001). Administración (11 ed.). México: McGrawHill.

Larico, R. (2015). Factores motivadores y su influencia en el desempeño laboral de los trabajadores de la municipalidad provincial de San Ramón-Juliaca 2014. (Tesis de maestría) Juliaca: Universidad Andina Nestor Cáceres Velásquez.

Lau, J. (2010). Evaluación del desempeño de personal bibliotecario. Buenos Aires: Alfagrama.

Martí, I. (2005). Diccionario enciclopédico de la Educación. Barcelona: CEAC.

Nickols, F. (2007). Performance appraisal weighed and found wanting in the balance. Obtenido de http://web.ebscohost.com/ehost/ pdf?vid=5\& hid=106\&sid=e768386f-b1684032-9f1a-3a6360 116d7c \%40SRCSM1

Nolberto, M. (2017). Gestión del talento humano y el desempeño laboral de la Municipalidad distrital de Pichanaqui, 2017. (Tesis de licenciatura) Huánuco: Universidad de Huánuco. 
Paz, C., \& Gonzales, D. (23 de Agosto de 2013). Productividad y competitividad. Obtenido de Alfa y Omega: http://nulan.mdp.edu.ar

Pozner, P. (2000). Módulo 2: Competencias para la profesionalización de la Gestión Educativa. En Gestión Educativa Estratégica. Buenos Aires: Editorial IIPE.

Quispe, E. (2015). Clima organizacional y desempeño laboral en la Municipalidad distrital de Pacucha, Andahuaylas, 2015. (Tesis de licenciatura) Andahuaylas: Universidad Nacional José María Arguedas.

Robbins, S. (2004). Comportamiento Organizacional. México.

Sistema Nacional de Evaluación, Acreditación y Certificación de la Calidad Educativa. (23 de Mayo de 2006). Ley No 28740. Obtenido de http://ctmperu.org.pe/documentos/LeyN-28740.pdf

Synthesis. (2008). La gestión escolar en las instituciones educativas. Obtenido de www.uach.mx/extension_y_difusion/synthe sis/2008/11/10/Gestios_escolar.pdf

Tapia, G. (2003). Un plan para la mejora de la gestión de la escuela. México: Editorial Rueda.

Torres, E. (Enero-Junio de 2015). Gestión educativa y su relación con la práctica docente en las instituciones educativas emblemáticas de la ciudad de Puno-2014Perú. Scielo Perú, 6(1), 56-64.

Uría, D. (2011). El clima organizacional y su incidencia en el desempeño laboral de los trabajadores de Andelas Cía. Ltda. de la Ciudad de Ambato. (Tesis de titulación) Ambato: Universidad Técnica de Ambato.

Valcarcel, G. (2009). Experiencias e Innovación. Salamanca: Davinci.

Vigo, A. (2012). Comparación de los sistemas educativos Latinoamericanos. (Tesis de doctorado) Lima: Universidad Wiener.

Werther, W., \& Davis, L. (2000). Administración de personal y recursos humanos. México: McGraw-Hill.

Yábar, I. (2013). La gestión educativa y su relación con la Práctica Docente en la Institución Educativa Privada Santa Isabel de Hungría de la ciudad de Lima-Cercado. (Tesis de maestría) Lima: Universidad Nacional Mayor de San Marcos.

Zhunio, J. (2016). Nuevo modelo de gestión educativa.

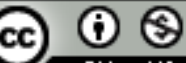

EY NC Esta obra está bajo una licencia de Creative Commons Reconocimiento-No Comercial 4.0 Internacional. Copyright (c) Martha Nancy Mosquera Maldonado 
